\title{
TECHNOLOGY AND EQUIPMENT FOR ELECTRON BEAM WELDING OF STRUCTURES IN AEROSPACE INDUSTRY
}

\author{
V.M. NESTERENKOV and K.S. KHRIPKO \\ E.O. Paton Electric Welding Institute, NASU \\ 11 Kazimir Malevich Str., 03680, Kiev, Ukraine. E-mail: office@paton.kiev.ua
}

\begin{abstract}
Electron beam welding is one of the leading technological processes used in development of structures for aerospace industry. For many years PWI has taken leading positions in development of specialized equipment for EBW. The paper provides description of different types of developed units, differing by overall dimensions of welding chamber, as well as variants of chambers and configurations of vacuum systems, displacement mechanisms of the EB gun or item being welded. All the units are fitted with modern systems of control of equipment included into them. Samples of aerospace products manufactured by EBW are given. 6 Ref., 16 Figures.
\end{abstract}

Keywords : electron beam welding, aerospace industry products, units, welding chambers, chamber overall dimensions, vacuum system, displacement mechanisms, control systems, welded components

Aviation and space industries occupy a leading position as to application of light and strong alloys of non-ferrous metals. Considering the priority of these directions, PWI paid a lot of attention to development of technologies of welding such materials by different processes, including EBW [1, 2].

Application of computer numerical control (CNC) tools for EBW brought this technology into the category of leading processes, owing to the capabilities of precision control of both electron beam movement trajectory in welding and its energy, including regulation of the total value of power and shape of this power distribution in space $[3,4]$. This ultimately led to an essential expansion of EBW technological capabilities and to improvement of repeatability of the process of welding batch-produced parts in the programmed mode.

Main characteristics of PWI developed units are as follows [5]:

1. «Small» (small-sized) units have up to $0.26 \mathrm{~m}^{3}$ volume of welding (vacuum) chamber (Figure 1).

Working pressure in EB gun is less than $5 \cdot 10^{-5}$ Torr, and not higher than $(2.5-3.0) \cdot 10^{-4}$ Torr in the welding chamber. Typical time of pumping down the welding chamber and gun is equal to not more than $5 \mathrm{~min}$ (depends on vacuum system components).

The units are of a comparatively simple design with permanently fixed outside welding gun. Welding movement is realized by miniature CNC mechanism of displacement of the part being welded: one- and two-coordinate work table and welding manipulator (rotator), mounted in one of the two positions - either with vertical axis of rotation of the faceplate or with horizontal axis. Moreover, the unit can be fitted with, for instance, miniature tailstock (for horizontal orientation of the axis of rotation of the welded part), small lathe chuck, additional center, etc.).

This type of units is fitted with a comparatively low-power high-voltage welding power unit of 6-15 kW (at fixed accelerating voltage of $60 \mathrm{kV}$ ).

These are versatile units, typical purpose of which is welding small-sized parts with linear and circumferential welds from different structural metals, including aluminium, magnesium and titanium alloys, usually of less than $30 \mathrm{~mm}$ maximum thickness.

2. «Medium» (midsized) units have welding (vacuum) chamber of 2.7-5.7 $\mathrm{m}^{3}$ volume.

Working pressure in EB gun is less than $5 \cdot 10^{-5}$ Torr, and not higher than $(2.5-3.0) \cdot 10^{-4}$ Torr in welding chamber. Typical time of pumping down the welding chamber and the gun is not more than 12-15 $\mathrm{min}$.

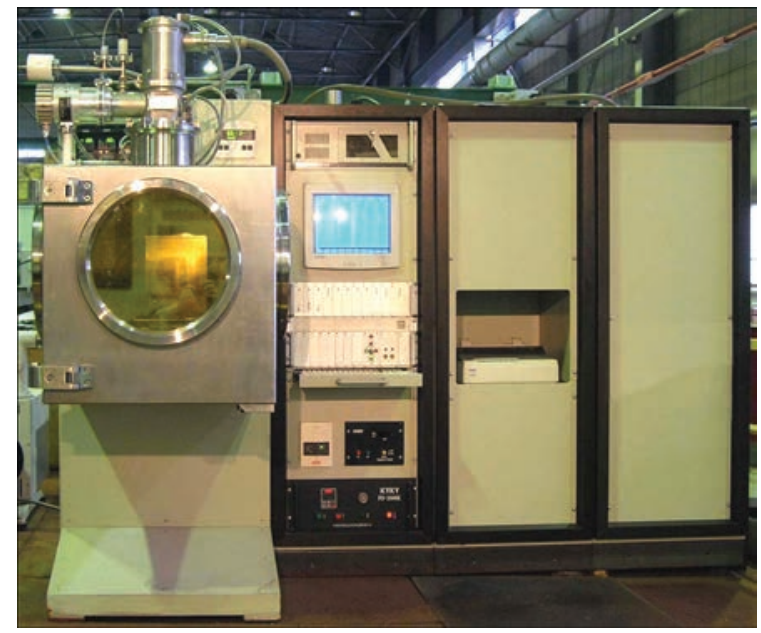

Figure 1. Small-sized unit for EBW 


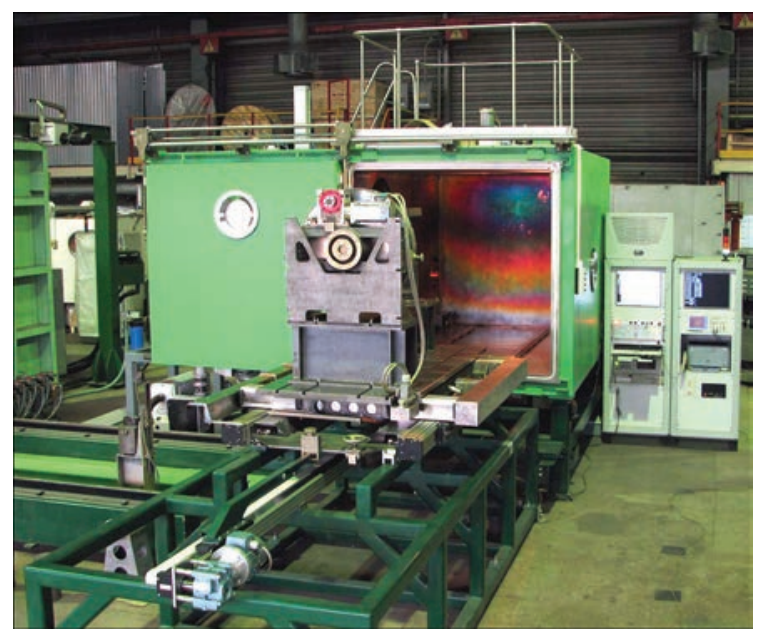

Figure 2. Medium-sized unit with stationary EB gun and two-coordinate work table

Such units have external permanently fixed (vertical) welding gun (Figure 2).

Welding displacement $(\mathrm{CNC})$ is here realized by precision two-coordinate work table and welding manipulator (rotator), mounted on work table top, either in the position with vertical axis of faceplate rotation, or with horizontal axis (Figure 3). Displacement of the entire moving base of the work table (on rails) from vacuum chamber onto loading-unloading platform and back into the chamber, with smooth approach to the parking position in the chamber proper, is additionally realized.

The unit can be fitted with tailstock with rotating or non-rotating center paired with the main welding manipulator (at horizontal orientation of its axis of rotation), as well as lathe chuck and additional center for this welding manipulator, etc. In addition, the units are usually fitted with paired set of special cabinets-stands for welding manipulator and tailstock, respectively (see Figure 3). Stands for the same manipulator, but for the case of vertical orientation of the axis of its rotation can be additionally supplied. In the general case, presence, quantity and height of stands

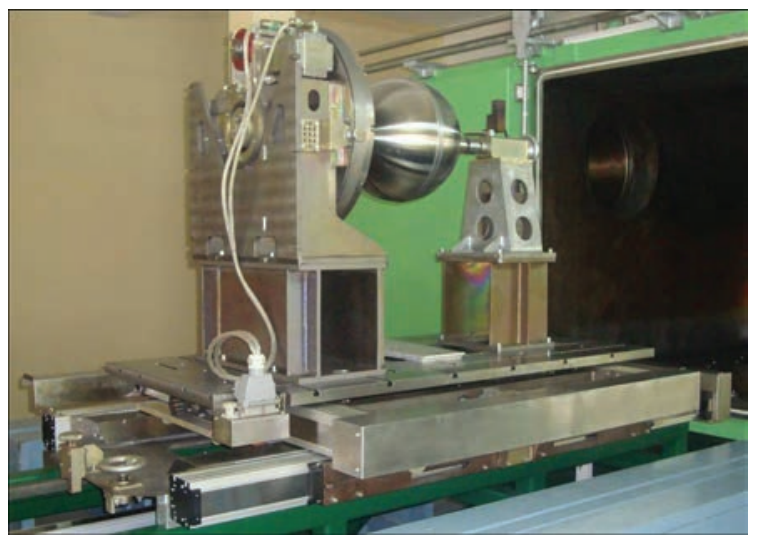

Figure 3. Two-coordinate work table of medium-sized unit with welding manipulator (rotator) mounted on special stands and tailstock

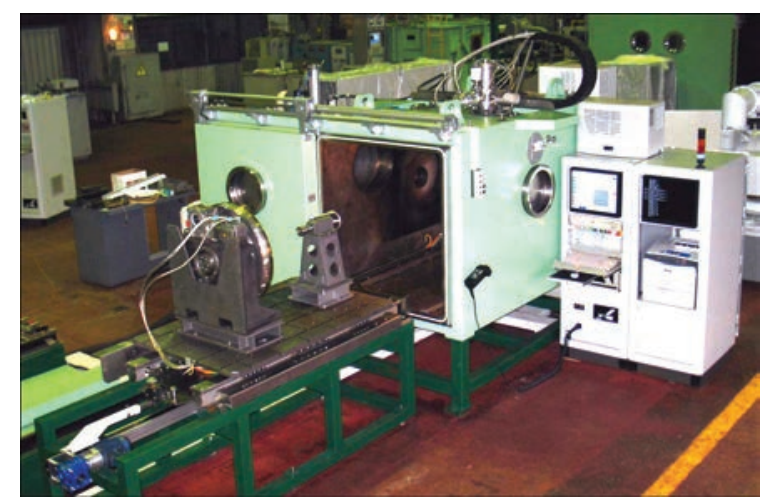

Figure 4. Medium-sized unit with mobile welding gun and one-coordinate work table

are selected, proceeding from the maximum admissible value of working distance from EB gun to outer surface of parts of a specific typesize.

In some cases, by customer request, combined schematic of linear welding displacement is used in midsized units (Figure 4): mobile EB gun module, moving along welding chamber roof along one axis (usually, $Y$ axis) and one-coordinate work table, moving along the other - transverse axis (axis $X$, respectively).

Irrespective of the above-mentioned schematic of welding displacement, typical linear working travel of such units is about $500 \mathrm{~mm}$ along both axes $X$ and $Y$. Such working travel along axis $X$ is due to sufficiently large length of work table top, capable of accommodating welding manipulator and tailstock along its length, with welded item fixed between them.

Units of this type are usually fitted with high-voltage welding power unit of 15 or $30 \mathrm{~kW}$ (at fixed accelerating voltage of $60 \mathrm{kV}$ ).

Such units, similar to «small» ones, can be used for welding all kinds of small-sized parts with linear and circumferential welds from any structural metals, including also aluminium, magnesium and titanium alloys of up to $60 \mathrm{~mm}$ maximum thickness. Nonetheless, their main purpose is welding cylindrical and spherical parts of up to $700 \mathrm{~mm}$ diameter. So, in particular, these units perform welding of billets of hemispheres of spherical tanks of various typesizes from structural titanium alloy Ti-6Al-4V and their subsequent final joining by through-thickness circumferential weld (see Figure 3).

3. "Large» (large-sized) units (Figure 5) have welding (vacuum) chamber of 19 to $42 \mathrm{~m}^{3}$ volume.

Working pressure in EB gun is less than $5 \cdot 10^{-5}$ Torr, and in the welding chamber it is not higher than (2.5$4.0) \cdot 10^{-4}$ Torr. Time of complete pumping down (i.e. both welding chamber and gun) is not more than 2040 min, depending on vacuum system components.

Unit configuration envisages mobile in-chamber welding gun, precision CNC displacement mechanism of which ensures linear movement along three 


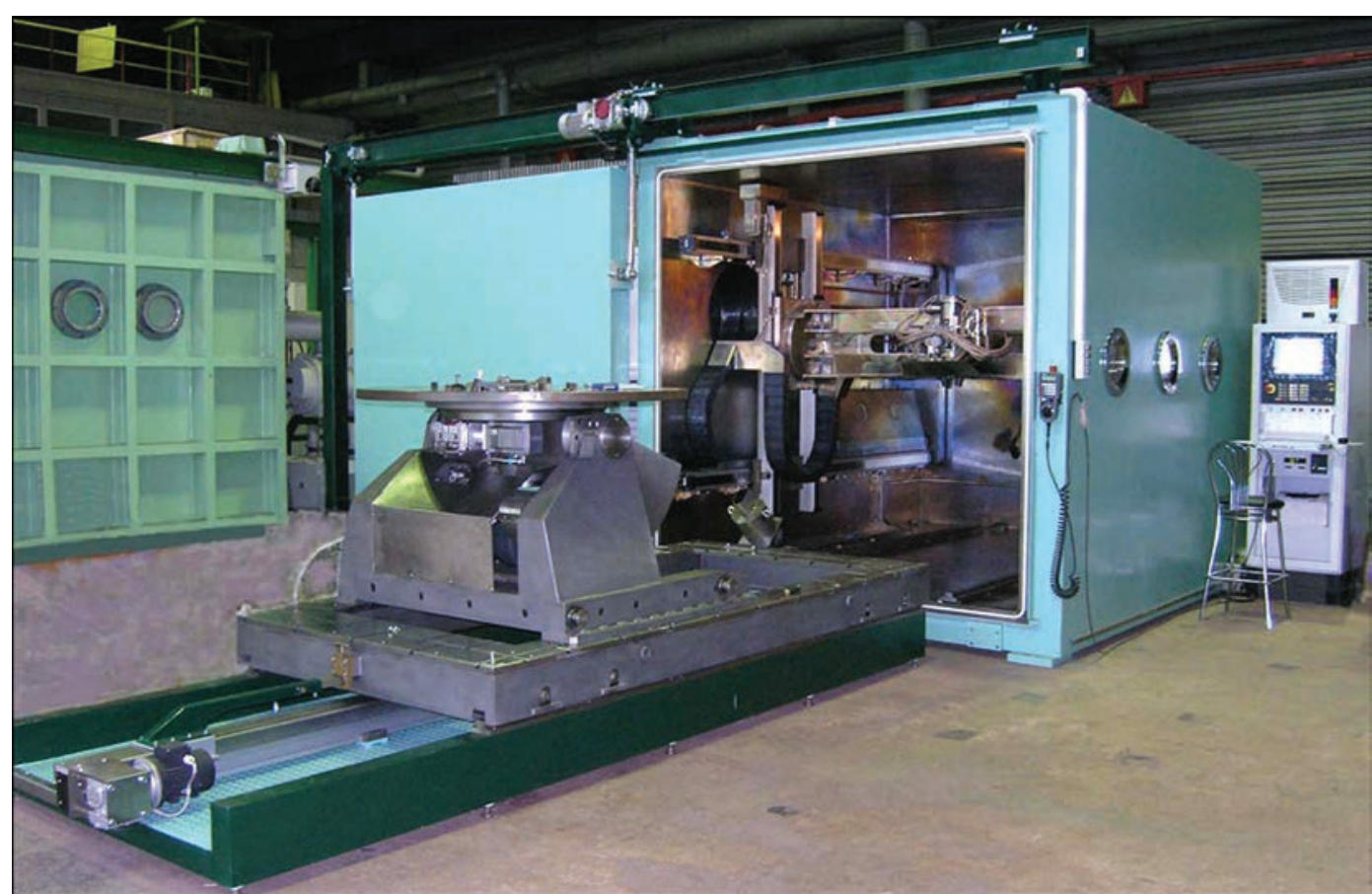

Figure 5. Large-sized unit with mobile in-chamber welding gun and work table (roll-out carriage)

coordinate axes (along the chamber $-X$, across the chamber $-Y$, and vertically $-Z$ ), as well as gun inclination up to $90^{\circ}$ in $Z-X$ plane (from gun «vertical» orientation to its «horizontal» orientation).

Unlike earlier described smaller units, work table in «large» units does not participate in the movement and is a loading-unloading carriage, with upper mounting plate with T-shaped slots, designed for placing and fixing various types of welding manipulators and additional accessories to them, as well as for all kinds of specialized welding fixtures for non-rotating parts and components. Naturally, there is the respective mechanism of work table movement on rails from the chamber to external loading-unloading platform, and back into the welding chamber with smooth approach to parking position.

A feature of «large» unit design is the fact that the above-mentioned gun rotation is realized through rotation of the base of cantilever beam, carrying the mechanism of displacement along axis $Y$ (Figure 6). Here, this rotation assembly proper can move freely within the greater part of welding chamber in $Z-X$ plane.

Depending on the unit purpose, the welding gun module can have an additional degree of freedom: CNC-axis of gun rotation by $\pm 45^{\circ}$ in a plane parallel to axis $Y$.

Rotation of the part being welded is usually ensured by one of three standard welding manipulators: welding manipulator with horizontal axis of rotation (it is usually supplied with tailstock of respective height), welding manipulator with vertical axis of rotation (both the manipulators can be replaced by one all-purpose manipulator, designed for both the orientations (horizontal and vertical), and welding manipulator with inclined axis of rotation. The latter allows inclination of the axis of faceplate rotation in the range from -30 up to $+90^{\circ}$ (from the vertical), that enables handling, for instance, complex sections of aircraft engines (Figure 7), or other aircraft components, for instance, aircraft wing pylon (Figure 8).

To expand production capabilities, welding chambers in «large» units can have not one, but a pair of «sliding» working doors and a pair of mobile work tables, respectively.

This type of units is fitted high-voltage welding power unit of 15, 30 or $60 \mathrm{~kW}$ (at fixed accelerating voltage of $60 \mathrm{kV}$ ), depending on specific materials being welded and their thicknesses.

Such units can be called «conditionally all-purpose» as they, in principle, can be used for welding most of the components fitting into in-chamber di-

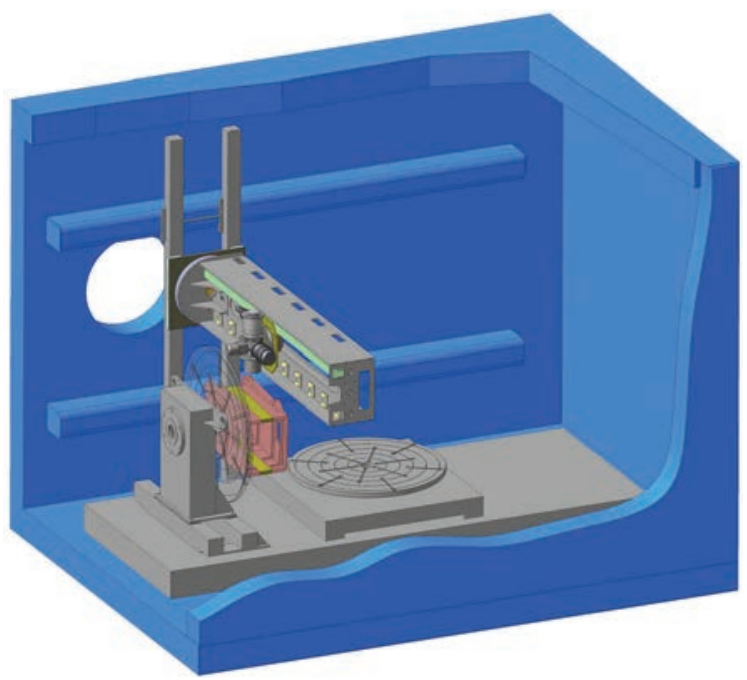

Figure 6. Model of in-chamber mechanisms of typical large-sized unit for EBW 


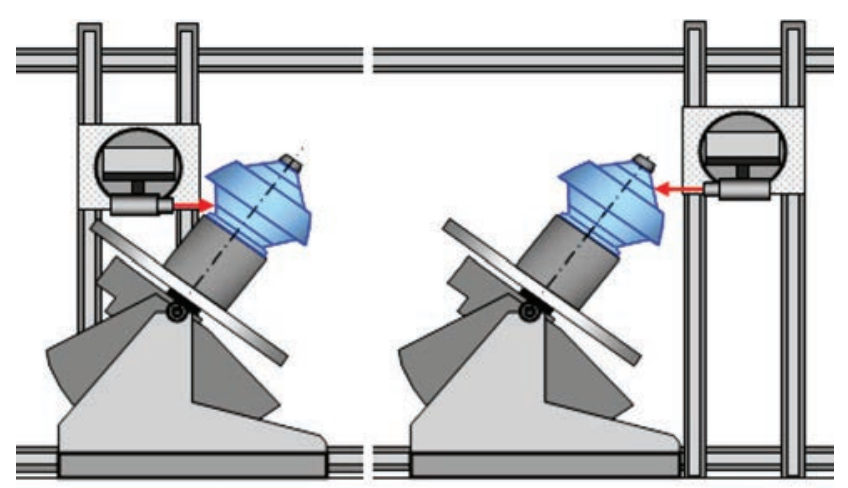

Figure 7. Example of EBW schematic of rotating parts of complex shape using welding manipulator with inclined axis of rotation

mensions, welded thicknesses of which are within the capabilities of the supplied power unit. However, their typical purpose is welding components of aircraft engines and other comparatively compact parts and components [6] (Figures 9-12).

4. «Superlarge» (particularly large-sized) units have welding (vacuum) chamber volume of 80 to $100 \mathrm{~m}^{3}$.

As to their purpose, such units (Figure 13) do not, in principle, differ from «large» ones and can also be regarded as «conditionally all-purpose», but they are designed for components of much larger dimensions, respectively. The range of thicknesses being welded still is within the limits of capabilities of similar welding power units of 15,30 or $60 \mathrm{~kW}$ (at fixed accelerating voltage of $60 \mathrm{kV}$ ).

Thus, the purpose of such units is welding largesized components of aircraft engines and other largesized and long parts.

Working pressure in EB gun is below $5 \cdot 10^{-5}$ Torr, and not higher than $(2.5-4.0) \cdot 10^{-4}$ Torr in the welding chamber. Time of pumping down the welding chamber and the gun is not more than 20-40 min (it also depends on the set of vacuum system components and on required working vacuum).

To ensure such comparatively short time of pumping down, this type of units are fitted with efficient vacuum systems (Figure 14).

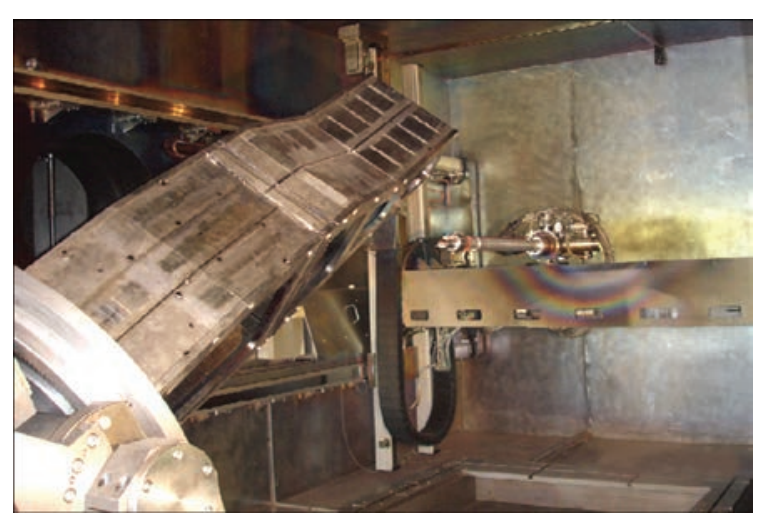

Figure 8. Pylon of aircraft wing on welding manipulator with inclined axis of rotation

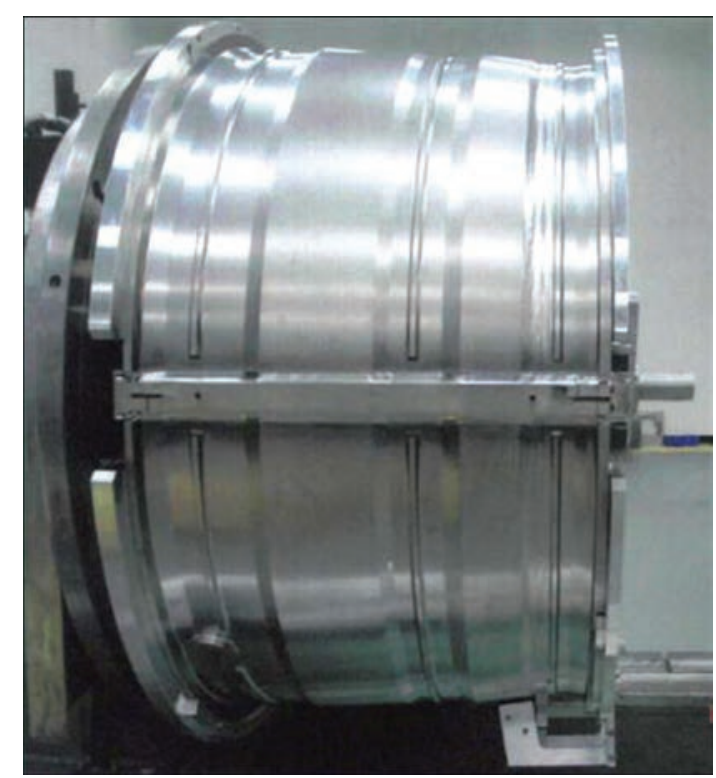

Figure 9. Billet of section of aircraft engine from titanium alloy

Configuration of such units further incorporates mobile in-chamber welding gun, precision CNC displacement mechanism of which ensures similar linear motion along three coordinate axes (along the chamber $-X$, across the chamber $-Y$, and vertically $Z$ ), as well as gun inclination up to $90^{\circ}$ in $Z-X$ plane. This gun inclination is also realized through rotation of the entire mechanism of displacement along $Y$ axis. However, unlike «large» units, in «superlarge» units gantry design is usually used instead of cantilever design. Here, the parts of rotating mechanism of the beam of $Y$ axis (i.e. angle of gun inclination in $Z-X$ plane) are fixed between two symmetrical gantries, which ensure free movement of the gun in greater part of welding chamber in $Z-X$ coordinate plane. Depending on the unit purpose, it can additionally be fitted with CNC-axis of gun rotation through $\pm 45^{\circ}$ in the plane parallel to $Y$ axis.

Similar to «large» units, welded component rotation is ensured by one of three standard welding ma-

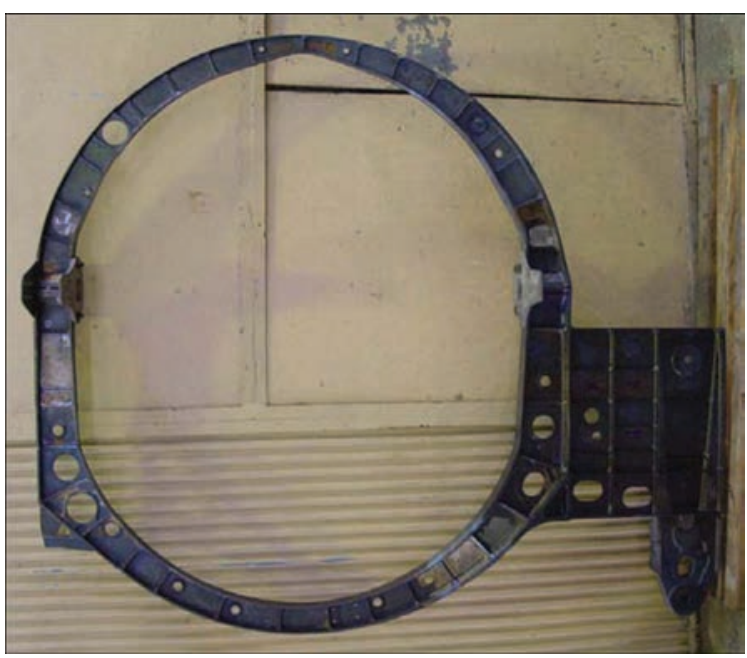

Figure 10. Billet of aircraft component of titanium alloy butt welded from several sections 


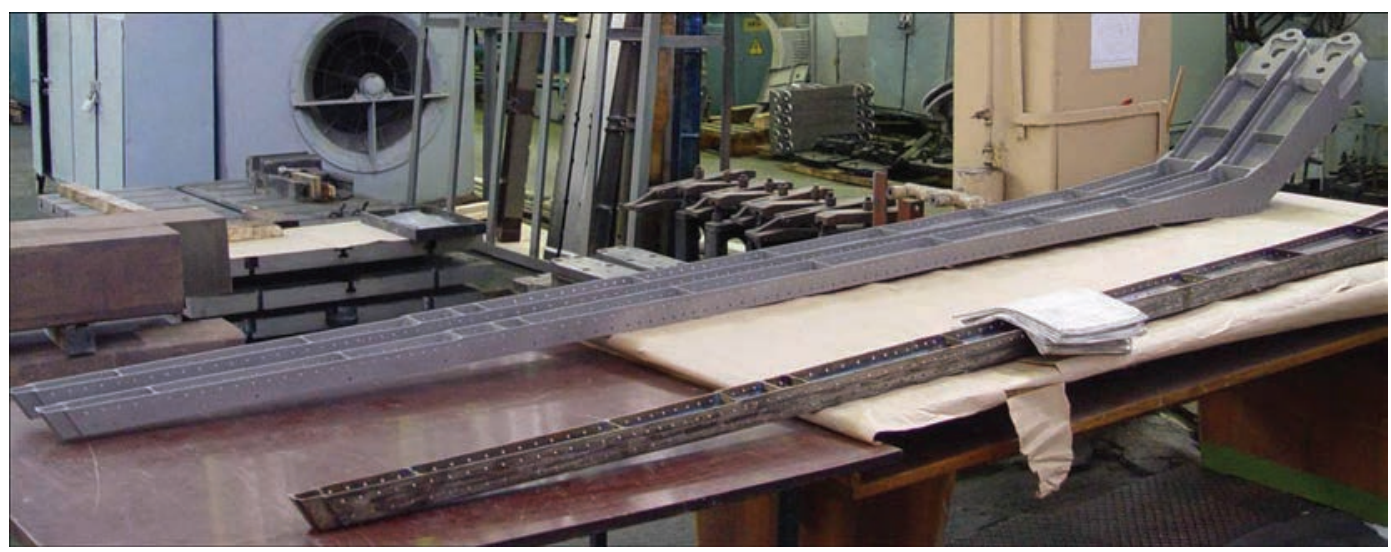

Figure 11. Long aircraft structures welded by electron beam
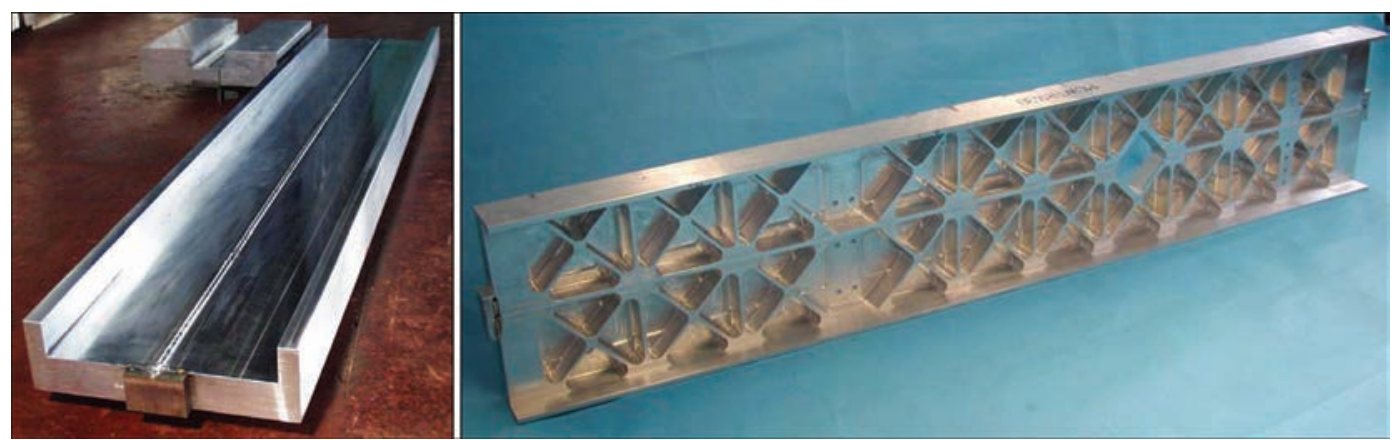

Figure 12. Welded blank (left) of aircraft wing beam from high-strength aluminium alloy (right — beam after machining)

nipulators: welding manipulator with horizontal axis of rotation (usually paired with the respective tailstock), welding manipulator with vertical axis of rotation, and welding manipulator with inclined axis of rotation.

All types of units are fitted with modern high-voltage inverter welding sources of respective power (set up for fixed accelerating voltage of $60 \mathrm{kV}$ ). The source was specially developed for highly efficient and reliable EBW: minimum response time of control circuits of accelerating voltage and welding current (electron beam current) has markedly improved their stability.

High-voltage inverter power unit is used as the source of $60 \mathrm{kV}$ accelerating voltage. The other part of welding source, including filament and bias modules, as well as stabilizer crate, incorporates all the recent PWI developments in the field of EBW control. As a result, all key channels (accelerating voltage channel, channels of filament and bombardment of

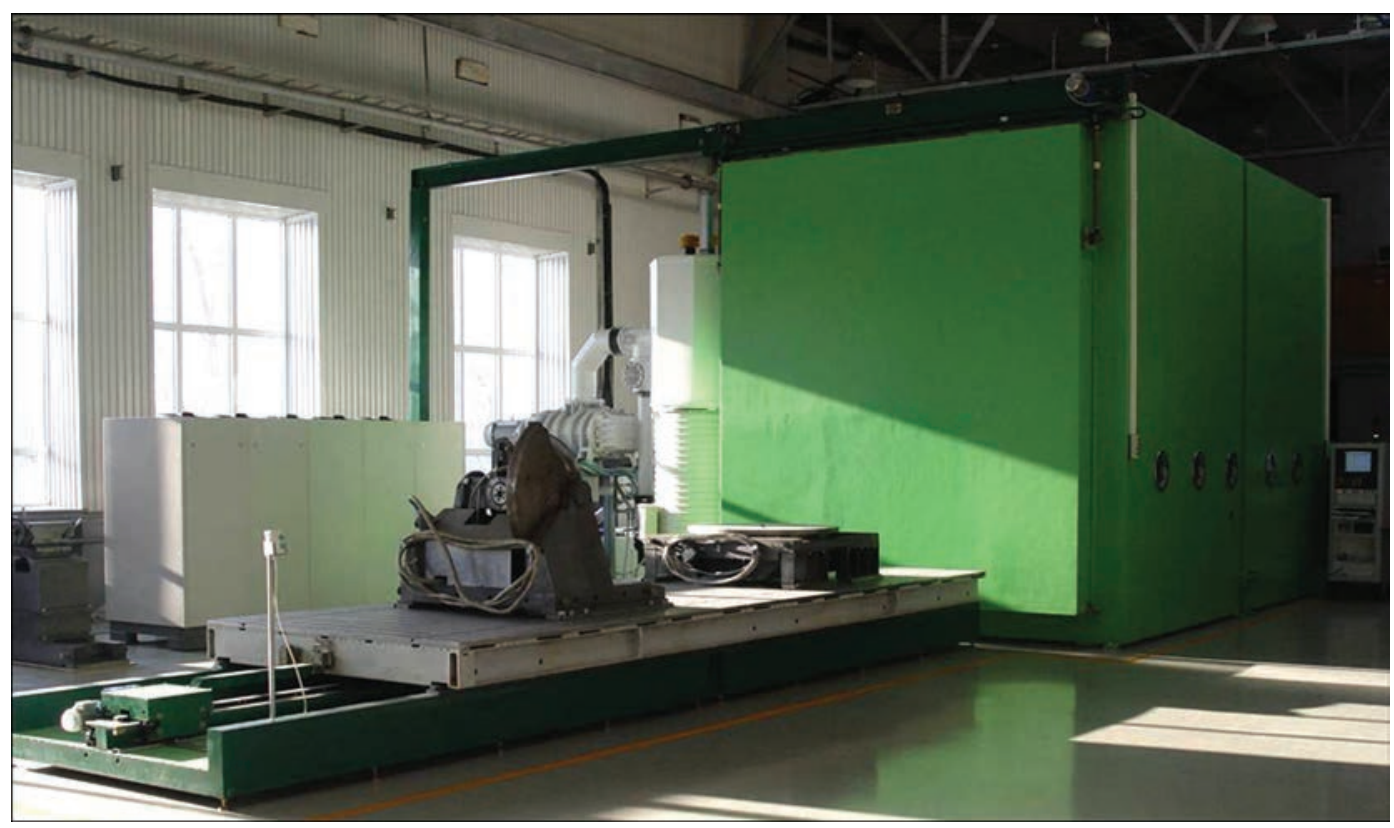

Figure 13. Appearance of superlarge unit fitted with mobile in-chamber welding gun and two work tables (loading-unloading carriages). Chamber is fitted with two doors 


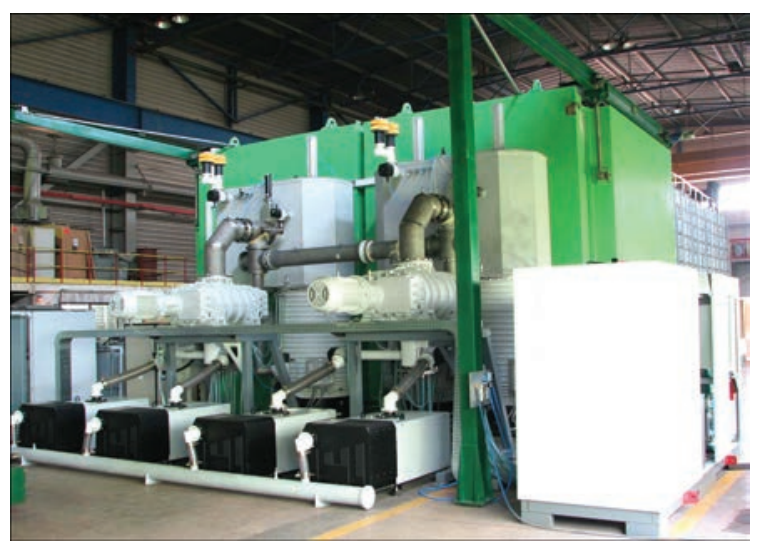

Figure 14. Vacuum system of welding chamber of $100 \mathrm{~m}^{3}$ volume welding cathode) have high-frequency power, formed on the basis of signals of individual microcontroller, operating by the respective algorithm. In terms of design all the high-voltage parts of the source are located in a special oil tank (Figure 15, position $a$ ), and low-voltage boards are in stabilizer crate (position $b$ ) and in the module (modules) of high voltage control (position $c$ ). Differing «earth» levels are galvanically decoupled and have separate power sources. Setting and feedback connection of different levels is performed through fiberoptic lines.

Welding source is quite compact in size and at up to $30 \mathrm{~kW}$ power inclusive, it usually fits into one power cabinet, to which power and interface cables are connected, and from which just the power cable comes out (usually through the roof), which powers the gun. In the case of $60 \mathrm{~kW}$ power the welding source usually consists of two parts.

Welding source is a hardware complex, all interaction with which proceeds through industrial interface (CAN bus). The only exception is special modulation signal from RASTR system, fed in the initial form. This is realized due to external connections being provided by respective microcontroller modules, connected to CAN bus and designed for control and diagnostics of all the welding source channels. These modules, in their turn, directly interact with actuator elements of the welding source, including the commercially manufactured accelerating voltage source.

Together with the welding source, all the units are fitted with RASTR- 6 system of secondary-emission electron imaging, integrated into this source. Such integration is due to the fact that functioning of this system is directly related to formation of the electron source. The integration results in the welding source momentarily forming, on the base of modulation signal generated by RASTR- 6 system, an electron scan pattern on the welded part surface by a low-power («probing») electron beam, controlled by microcontroller of welding current channel by a special law.

At passing of "probing» electron beam along the scan pattern lines, secondary electrons are emitted in

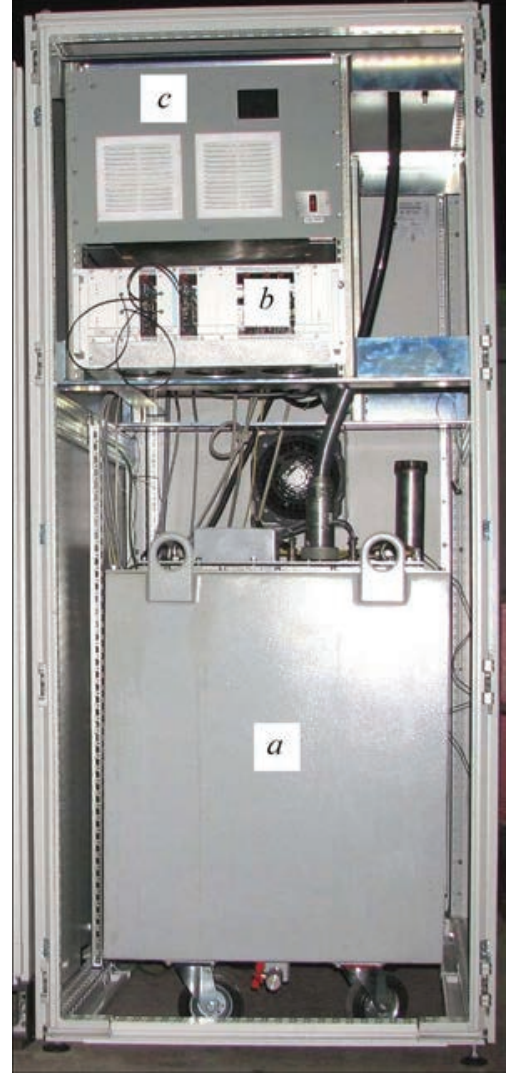

Figure 15. Cabinet of welding source of $15 \mathrm{~kW}$ power

the site of point bombardment of the part surface by beam electrons (primary). These electrons are captured by special passive sensor (Figure 16, a) usually located on welding gun end face, which can be of different design (usually depending on application area, i.e. it can be adapted to suit the configuration of welded parts). Located directly near this sensor, is a compact module of preamplifier, which forms and enhances the useful signal, the voltage of which is proportional to current value read from the sensor. This, already amplified signal, is taken out of the chamber through a shielded line and comes to the system main video amplifier. Ultimately, the signal is digitized by specialized computer board WLCA and is output to the interface in the form of an image (Figure 16, $b$ ) used both for visual observation and for manual guidance to the butt being welded, and for operation of special program algorithms helping the user (welding operator) to make new welding programs and reproduce the already existing programs in welding of recurring typical parts. The system allows forming a quite stable image of the welding zone, both before the start of welding and after its completion, and directly during performance of welding proper.

RASTR-6 system design incorporates microcontroller module of forming process scan patterns, controlled by CAN bus, as is the entire welding source. As a result, scan signals generated by this module in both the channels (deflecting coil poles) are enhanced 

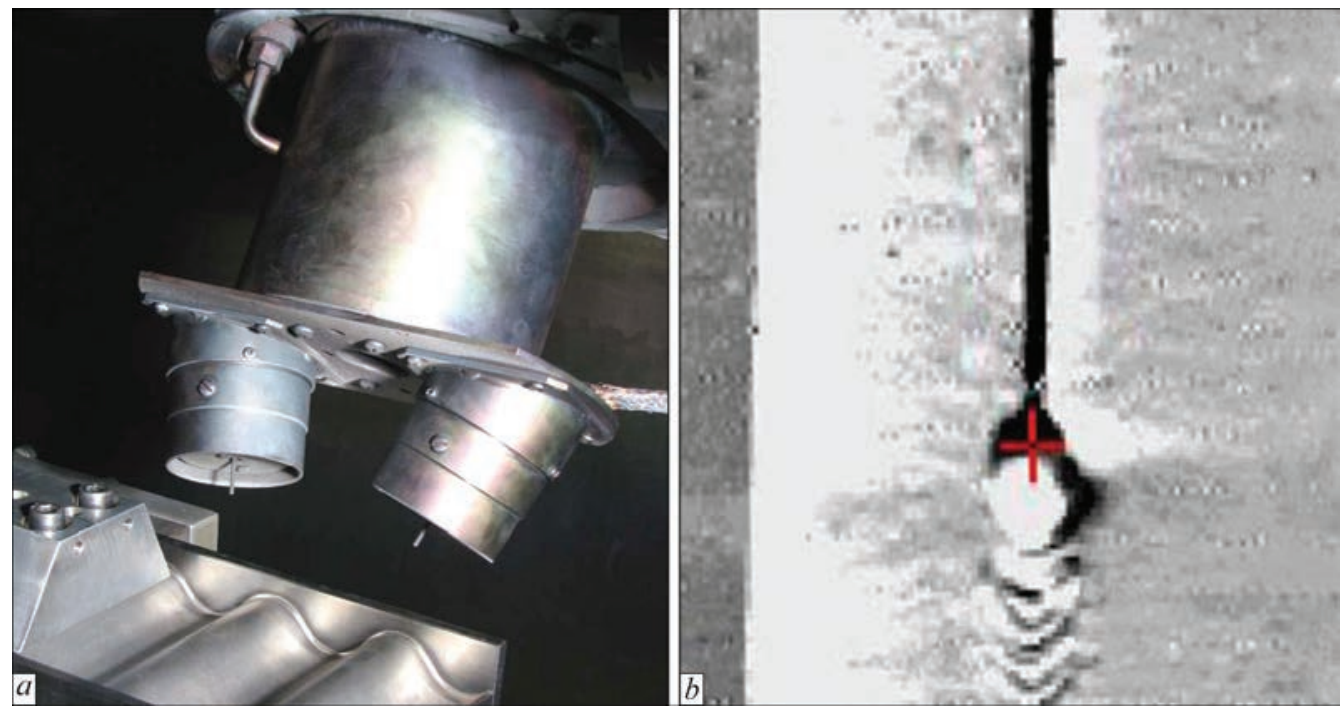

Figure 16. Sensor of secondary electrons of RASTR-6 system (a), and welding zone image formed by it $(b)$

by standard amplifiers of line and frame scanning of RASTR-6 system.

All EBW units, developed and manufactured at PWI, have modern system of controlling all the equipment included into their structure. The main part of the equipment, on which the welding process proper depends, is under continuous program control, providing both fully automated and manual mode of this equipment functioning. Here, the concept of high-level program control is used. User interacts with the equipment solely through Windows-oriented graphic interface (GUI), which is operated using standard tools: keyboard and mouse-type manipulator. Interface was developed so as to be intuitively clear to any person, who has already used software in Windows $\mathrm{OE}$, and requires absolutely no special qualifications for operating low-level machine programming (G-codes, etc.). Each of equipment subsystems has the respective window graphic tools with the required checking of input data and locks, primarily, to ensure the safety of the user himself and of equipment operated by him. The program has all the tools for controlling the vacuum system, manual displacement of welding gun and part, manual control of welding parameters, compiling automatic welding programs (welding by a program), their storage and fulfillment, as well as tools for acquisition and storage of diagnostic information on operation of key subsystems of the unit, administrative tools, etc.

Hierarchical control system was divided into two hardware levels. Upper level includes all the tools for interaction with the user, including graphic interface, tools for welding program compiling and storage, diagnostic data acquisition and storage, administering, etc. Lower level directly supports fulfillment of all the procedures by commands from the upper level.

At present PWI uses two basic configurations of the system of EBW unit program control. The first envisages application of Siemens complete industrial system (CNC + PLC) Sinumeric 840D, and the second one - application of trimmed industrial system Synamics S120, also of Siemens company.

In the case of application of complete Sinumeric $840 \mathrm{D}$ system, the main user interaction program is installed in standard upper level industrial computer Sinumeric PCU-50, operating in Windows environment.

Profinet system is used to transmit data from its upper level to lower software and hardware level, which includes: machine control panels Sinumeric MCP, Sinumeric NCU, connecting module Basic PN, to which mobile manual terminal Sinumeric HT 2 is connected (it is used as remote panel to control manual movement of the gun/part). In its turn, NCU, through network connection DRIVE-CLiQ, interacts with the modules of servomotors of welding displacement axes and their encoder modules, and through ProfiBUS bus it interacts with analog and digital input/output station Simatic ET 200M.

Computer servicing RASTR-6 system is an additional element of the lower level. Control program of this system is in constant interaction with upper level main program (in PCU-50), using Ethernet-connection. As a result, upper level program can at any moment display the secondary emission image of welded part surface, formed and transmitted by RASTR- 6 system. More over, at operation of specialized automated algorithms of «searching» for the butt being welded (when making the program of the trajectory of welding a new part and for correction of the existing trajectory in welding typical recurring parts) both the control programs of the upper level and of RASTR system operate inseparably.

Welding displacement (gun/part) is $\mathrm{CNC}$ controlled (Sinumeric NCU), both in manual displacement mode, and in automatic welding. In the latter case, the text script prepared on the upper level of 
welding program is transmitted to NCU, where CNC, by its own algorithms, performs complete calculation of the trajectory, interpolations, velocities and accelerations along each of the axes. In automatic welding, $\mathrm{CNC}$ provides complete synchronization of all the axes, both mechanical, and virtual, for which channels of welding and focusing currents, as well as of process scanning of the electron beam, are used. Here, control tasks of these virtual axes are read from DMP module of high-speed outputs of Simatic ET 200M station, and are converted to the protocol of CAN bus (from which high-voltage welding source is controlled).

Control of welding parameters in the manual mode is performed from upper level computer, for which it has the respective CAN bus adapter.

NCU programmable logic controller (PLC) controls the vacuum system, using analog inputs, as well as digital inputs and outputs of Simatic ET 200M station. In the automatic mode the user just issues (from upper level) the commands for switching the respective operation mode of vacuum system («Pumping down», «Air», «Waiting» or «Stop»), the other decisions being taken by PLC, in keeping with the algorithm entered into it. Contrarily, in the manual mode, the user can control each vacuum system element directly (through the respective functions of the same PLC algorithm). Such a mode is designed exclusively for adjustment or repair operations, as all the locks of automatic algorithm are switched off, and the user takes up full responsibility for correct operation of the system that is always potentially dangerous.

The described control system has been actually introduced into standard modern machine CNC complex, the reliability of operation of which is ensured by many-year stepped evolution of the entire complex. Here, it should be noted that as a control complex for a welding unit with high-level control, it is somewhat redundant in its functionality (particularly, as regards $\mathrm{CNC}$ capabilities) and is not quite rational in terms of structure. In the latter case, we mean that stationary control panel Sinumeric MCP is not used at all, and high-speed analog outputs are actually used for other purposes. More over, an additional computer is required to control RASTR-6 system.

The above considerations led to control system based on "complete» Sinumeric $840 \mathrm{D}$ complex being used only by special customer request. In all the other cases, in EBW units (developed at PWI) a specialized two-level control system is applied, which is based on two simple standard single-board industrial computers, using «trimmed» Synamics S120 complex as the actuating mechanism to control multiaxial welding displacement and vacuum system. One of the computers is designed for functioning of Windows program of upper level control, with which the user interacts, and the second operates as the lower level — as CNC and PLC simultaneously. Modern computer capability is quite sufficient for performance of both high-priority tasks, characteristic for $\mathrm{CNC}$, and for low-priority tasks, characteristic for PLC. Ultimately, one lower level program runs in the computer under control of real-time operating system QNIX. This program is responsible for control of multiaxial welding displacement and welding source (and for synchronizing their simultaneous operation), as well as control of vacuum system and RASTR-6 system.

Both the industrial computers have respective Ethernet-adapters for exchanging the data of upper and lower level programs, as well as respective CAN bus adapters. In addition, lower level computer has ProfiBUS bus communications board (adapter).

As a result, interaction of all the system elements is almost completely performed through industrial CAN and ProfiBUS buses. Lower level program, via ProfiBUS bus, controls the vacuum system through Simatic ET 200M station, and controls welding displacements through the main part of Synamics S120. Here, in the case of automatic welding (by a program) it synchronizes the movement with the change of welding parameters via CAN bus. Manual movement tasks are transmitted to lower level either from upper level program, or from remote panel via CAN bus. Otherwise, control of both the welding source and RASTR-6 system is similar to the first considered variant.

PWI is in the process of continuous improvement of EBW equipment control system, enhancing the convenience and functionality of the developed equipment, taking into account the steadfast evolution of the hardware and component base, as well as accumulated many-year experience of development of the technology and equipment for EBW of diverse materials and their thicknesses.

1. Nazarenko, O.K., Kajdalov, A.A., Kovbasenko, S.N. et al. (1987) Electron beam welding. Ed. by B.E. Paton. Kiev: Naukova Dumka.

2. Ilyushenko, R., Nesterenkov, V. (2006) Novel technique for joining of thick section difficult-to-weld aluminium alloys. In: Proc. of $10^{\text {th }}$ Int. Conf. on Aluminium Alloys (Vancouver, Canada, July 9-13, 2006), Vol. 519-521, 1125-1130.

3. Paton, B.E., Nazarenko, O.K., Nesterenkov, V.M. et al. (2004) Computer control of electron beam welding with multi-coordinate displacements of the gun and workpiece. The Paton Welding J., 5, 2-5.

4. Akopiants, K.S., Nesterenkov, V.M., Nazarenko, O.K. (2002) Electron beam welding of $60 \mathrm{~mm}$ thick steels using longitudinal oscillations of beam. Ibid., 9, 2-4.

5. Nazarenko, O.K. (2008) Up-to-date equipment of the E.O. Paton Electric Welding Institute for electron beam welding. Ibid., 10, 31-35.

6. Bondarev, A.A., Nesterenkov, V.M. (2013) Examination of weldability of MA2 magnesium alloy by electron beam in vacuum. Kompres. Mashinostroenie, 2, 21-28.

Received 15.03.2016 